\title{
Mathematical modeling and optimization of parameters of apodized Bragg gratings
}

\author{
MAKSAT KALIMOLDAYEV ${ }^{1}$, ALIYA KALIZHANOVA ${ }^{1,3}$, WALDEMAR WÓJCIK ${ }^{1,2}$, \\ GULZHAN KASHAGANOVA $^{1,4}$, SALTANAT AMIRGALIYEVA ${ }^{1}$, AZHIBEK DASIBEKOV $^{5}$, \\ AINUR KOZBAKOVA ${ }^{1,3}$, ZHALAU AITKULOV ${ }^{1}$ \\ ${ }^{1}$ Institute of Information and Computational Technologies CS MES RK, KAZAKHSTAN, \\ ${ }^{2}$ Lublin Technical University, POLAND \\ ${ }^{3}$ Al-Farabi Kazakh National University, KAZAKHSTAN \\ ${ }^{4}$ Kazakh-American University, KAZAKHSTAN \\ ${ }^{5}$ M.Auezov South Kazakhstan State University, KAZAKHSTAN
}

\begin{abstract}
In the result of carried out researches with simulation usage there have been obtained the dependence of the spectral reflectivity characteristics of Bragg gratings on the grating length and on the local changes of the fiber core refraction index, as well, that the spectral reflectivity thereof increases along with the grating length enlargement. Outcomes of mathematical modeling in MATLAB medium and carried out analysis of the light emission in the Bragg grating fiber structure clearly denote the possibility of time impulses control magnitudes extension. In the end it has been concluded, that we can precisely control the dispersive claddings in the fiber-optical line through appropriate selection of the Bragg fiber gratings apodization parameters.
\end{abstract}

Key-Words: - Fiber Bragg gratings, apodization function, fiber-optic sensors, mathematical model.

Received: February 14, 2019. Revised: January 31, 2020. Accepted: February 15, 2020. Published: March 25, 2020.

\section{Introduction}

Basis for wide prevalence of fiber sensors in the science and engineering is due to their high sensitivity and many unique properties: they are compact, simple in operation, they can brilliantly work under the conditions of severe electromagnetic interference, increased radiation, high temperatures, strain and under other adverse conditions. It shall be noted as well that the sensors themselves do not represent the source of any energy irradiation, i.e., can be applicable for any special usage. Fiber-optic sensors represent the optic fiber fragment, subjected to a certain modification. Use of optic fiber as the elements does not influence at the result of electric magnetic fields measurement, does not have side electromagnetic radiation, channels cross interference, there are no problems, connected with earthing network and voltage shift at the points of connection of dissimilar conductors, sufficiently increase electrical safety, no problems of arcing and flashing.

Substantial part of all fiber sensors comprise the so-called interferometric ones, operation principle of which is based on adding the fields of two electric magnetic waves. Registered intensity of their interference result depends on the phase difference. Considering that the optical waves length is infinitesimal, then measuring small phase shifts with a special equipment we can obtain the sensitivity to the change of interferometer beams path difference, which is unobtainable by any other techniques.

In interferometric sensors the change of the measured physical magnitude shall be transformed into the path difference of interferometric beams, which is fulfilled in the device's sensitive element.

Fiber interferometric sensors are divided into two classes: conventional (dual beam) and polirimetric. The first class sensors register the result of two colinear optical waves interference, outspreading along different fibers, at that, one fiber is bearing, another, subjected to the impact, is signaling. Such type of sensors is most widely distributed. It can be implemented on the basis of the known interferometric schemes, for instance, of Michelson, Macha-Tsender, Fabry-Pero (low-Q), Sagnac et alii.

Metering system on the basis of fiber-optic sensors allows implementing several dozens of sensors on a single fiber. As a fiber-optic sensor there is used an optical fiber with the recorded Bragg gratings along the fiber length. Using Bragg fiber gratings it is possible to construct a measuring system able to measure different magnitudes in real time scale.

Fiber gratings are actually the only type of sensor elements, which can be easily united into the arrays through multiplexing along the wave length [1]. For that purpose each sensor element is recorded according to its unique period, which 
allows differentiate them according to the spectrum upon analysis and accordingly, fix the showings of every sensor separately. Quantity of sensors in the massif is mainly limited by the source spectral width, amount of channels along the waves length and analyzer dynamic range. The main disadvantage of existing sensors is impossibility to define the bend direction.

The bend direction plays a great role, for instance, upon using the sensors for monitoring the conditions of wind turbines, situated far from human habitat, particularly at the seaside areas flooded with tide rises, building cranes, bridges, overpasses. Such monitoring systems allow transfer the data on the most important parameters to long distances in the real time mode.

\section{Bragg's apodized gratings}

Bragg fiber grating represents the Bragg mirror, namely, refraction index periodical structure mode, directly in the optical fiber core, with an approximate length of $1 \mathrm{~cm}$. Such structure reflects the light in the narrow spectral range. Gratings recording is carried out in special photo responsive optical fibers thanks to the so-called photorefractive effect [5]. There exist a lot of different means of constructing sensor systems based on Bragg gratings. In the simplest case the sensor system represents a point sensor, connected through optical splitter with a light signal source and analyzer block $[1,2]$. A signal from the source is reflected by a sensor element. Reflection wave length is fixed by an analyzer module. As a rule, an analyzer represents a narrow-band spectrometer. There are spectrometers of different types: from standard diffractive, where a diffraction grating is used as a dispersive element, to the analyzers based on FabryPero interferometer. By scanning the interferometer width we can analyze the sensor's refraction optical spectrum. Bragg wave length (central light wave length, reflected backward from the Bragg grating) depends on the grating period, which can change upon shifting or changing the temperature of constructions under control:

$$
\lambda_{B r}=2 n_{e f f} \Lambda
$$

where $n_{\text {eff }}$ - effective refraction index of the main mode fiber core $\Lambda$ - Bragg grating period.

Thus, according to the Bragg wave length change we can define the value of changing the denoted physical magnitudes of the constructions under control and assess their influence at constructions under control.

Sensors on the Bragg fiber gratings measure the strain in the definite point. However in one fiber we can record hundreds of Bragg fiber gratings and one analyzer will measure all reflections. It gives a chance to create very simple but effective devices which simultaneously control multitude of constructions different points. As the reflection wave length of the Bragg fiber grating is influenced by both temperature and deformation, the sensors are often placed in pairs, where one of them is isolated from the construction motion [4]. It allows independently measure both deformation and temperature.

There exist sensors, allowing simultaneously define the temperature and deformation (inner strain) using one grating [3]. In case herein the fiber-optic grating is recorded on anisotropic fiber. Due to anisotropy the effective refraction showings of orthogonally polarized modes differ from one another. As Bragg grating wave length depends on $n_{\text {eff, }}$ in anisotropic fiber the Bragg wave length of orthogonally polarized modes differ for a certain magnitude. At that, the difference thereof is up to the temperature, but does not depend on the strain change. Thus, according to shifting a separate Bragg wave length there is measured the strain change, and according to the difference change of Bragg lengths of orthogonally polarized modes - the temperature. As well, the long-period fiber gratings can be used as sensors. Long-period fiber gratings, in distinction from the Bragg gratings, do not reflect a signal, but they also have their unique transmittance spectrum at the expense of spectrally-selective scattering, depending on the grating period and fiber waveguide structure. In long-period grating the distance between the bars is much bigger than the wave length, and its spectrum is defined by the main and cladding modes interference.

Dependence of the transmittance spectrum on the long-period grating temperature comparing to the Bragg grating has critically another nature. Moreover, in relation to the fiber structure the change in the spectrum according to the temperature growth might be both positive and negative. Based on the phenomenon thereof there has been elaborated a relatively simple sensor system, which allows separating the loadings deformation component and temperature $[1,6]$.

As an example let's consider apodized Bragg fiber gratings.

Apodized Bragg's gratings. Bragg's homogeneous gratings spectrum, being an addition to the base peak, also has undesirable high indirect 
side lobes, which generate cross-interference between neighboring channels.

FBG definite applications impose certain requirements to the reflectance spectrum form, which is specified by the grating refractive index profile, one of the common requirements to them is absence of side lobes. The phenomenon thereof is undesirable, because it strives to wave liquidation.

Appearance of side lobes is connected with the fact that the Bragg grating structure, which has a finite length, quickly starts and finishes. It is of paramount importance to eliminate that side lobes reflective factor and bring it to the minimum. The most widely used method to eliminate the side lobes is the fiber Bragg gratings apodization. It means, that the reflection coefficient amplitude is modulated in such a manner, that it increases, but it also influences at the gratings dispersion characteristics. Apodization might be decreased gradually in compliance with the applied function. Thus, the apodization is a highly valued instrument for smoothing the Bragg grating reflection spectrum, reached by means of UV light action.

The ideal fiber Bragg's grating functioning is passing the beam crosswise, excluding passing the wave's one length infinitely thin. Only the wave's lengths, satisfying Bragg's condition will be reflected and spread out in opposite direction. Sure enough, transmission and characteristics of reflection are distorted by the so called side lobes. This phenomenon, as it is shown in the Figure 1, is undesirable, as it strives to the wave liquidation.

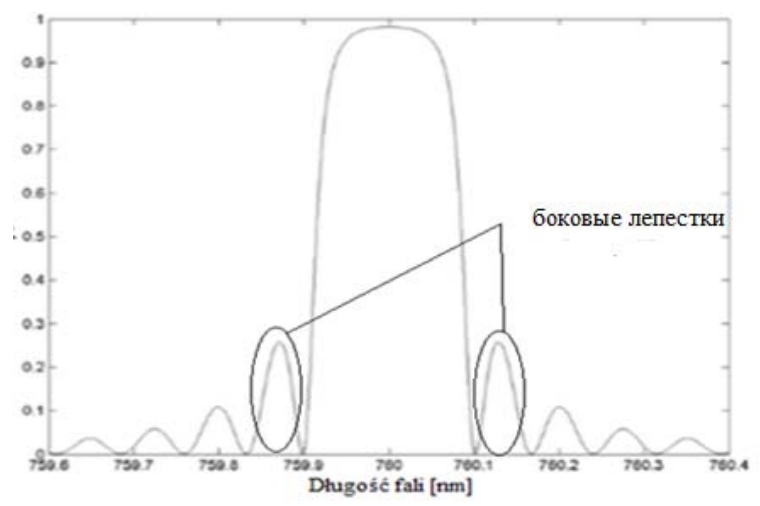

Fig.1 Reflection spectrum of fiber Bragg grating

It is very important to eliminate reflecting power of the side lobes thereof and lead them to the minimum. The most widely used method for eliminating the side lobes is fiber Bragg grating apodization [7]. It means, that refraction index amplitude is modulated in such a manner, that it grows and decreases in compliance with the applied function [8]. Thus, apodization is a valuable tool for smoothing the Bragg grating reflection spectrum but, as well, influences at grating dispersive characteristics. Apodization can be reached by means of UV-radiation impact.

Figure 2 shows the difference in sizes of the side lobes of fiber Bragg gratings reflection spectrum with apodization and without it.
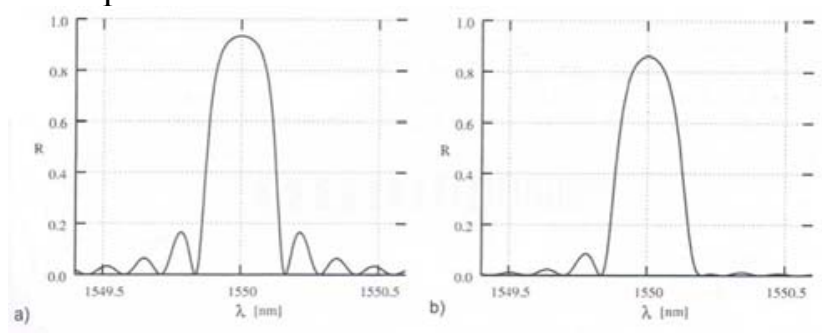

Fig. 2 Reflection spectrum of fiber Bragg grating a) without apodization, b) apodized

In case of apodized Bragg gratings the amplitude modulation increases and gradually decreases. Under amplitude modulation method the ups and downs speed occurs along the fiber axis and depends on the apodization function.

The technology thereof has opened new possibilities for creating and optimizing the fiberoptic elements, in particular, the filters, dispersion compensation modules, lasers with the distributed feedback and fiber-optic sensors for different parameters measuring. The most widely used method for eliminating the side lobes is the fiber Bragg gratings apodization.

Side lobes suppression is reached at the expense of securing the smooth modulation amplitude change and leveling the average value of the directed refraction index along the grating, the so called grating profile apodization.

There exist a lot of refraction index profiles, which allow receiving the FBG spectrum with suppressed side lobes [9], at that, practical implementation of most of them requires technologically complicated scanning techniques.

Apodization primary functions or apodization profiles and their formulae:

- Gauss function $g(z)=\exp \left[-a\left(\frac{z-\frac{L}{2}}{L}\right)^{2}\right]$;

- Raised sine function $g(z)=\sin ^{2}\left(\frac{\pi z}{L}\right)$;

- Sinc function $g(z)=\operatorname{sinc}^{X}\left(\left|\frac{2(z-L / 2)}{L}\right|^{Y}\right)$;

- Tanh function

$g(z)=1+\tanh [T(1-$

$\left.\left.2\left(\frac{z}{L_{g}}\right)^{\alpha}\right)\right]$ 
- Blackman functiong $(z)=0,42+0,5 \cos \left(\frac{\pi z}{a}\right)+$ $\frac{2}{25} \cos \left(\frac{2 \pi z}{a}\right)$;

- Hamming function $g(z)=\frac{1+H \cos \left(\frac{2 \lambda z}{l}\right)}{1+H}$;

- Cosine function $g(z)=\cos ^{A}\left(\frac{\pi}{L_{g}} z\right)$;

- Cauchy function $T(z)=\frac{1-\left(\frac{2 z}{L_{g}}\right)^{2}}{1-\left(\frac{2 B z}{L_{g}}\right)^{2}}$.

The most widely applied apodization functions are: Gauss function, Sinc function, Blackman function and Cosine function.

Let's consider the widely used apodization functions and their influence at spectral characteristics of fiber Bragg gratings.

Gauss function is expressed by the formula [9]:

$$
g(z)=\exp \left[-a\left(\frac{z-\frac{L}{2}}{L}\right)^{2}\right]
$$

\subsection{Numerical solution}

Examples of Gauss profiles for various parameters values $a$ are represented in figures 3, 4, 5, 6 .

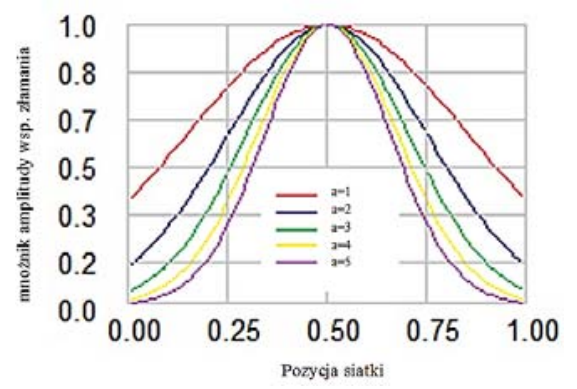

Fig. 3 Gauss apodization profiles for different values of parameter a

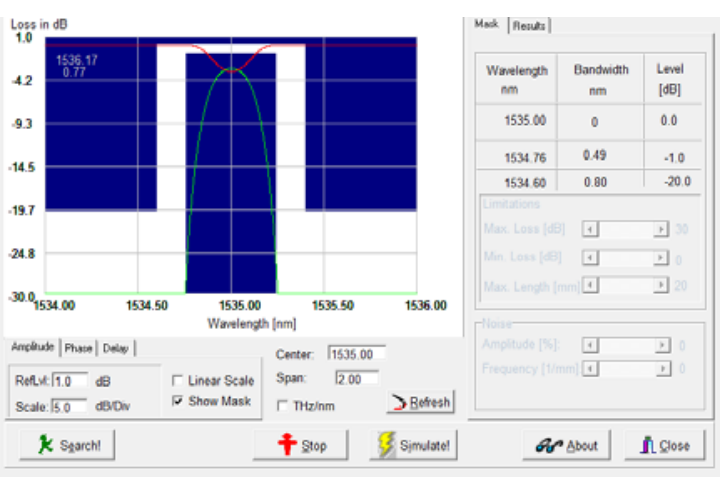

Fig. 4 Comparison of fiber Bragg gratings characteristics without apodization - blue line and apodized Gauss function at $\mathrm{a}=2$ - red line

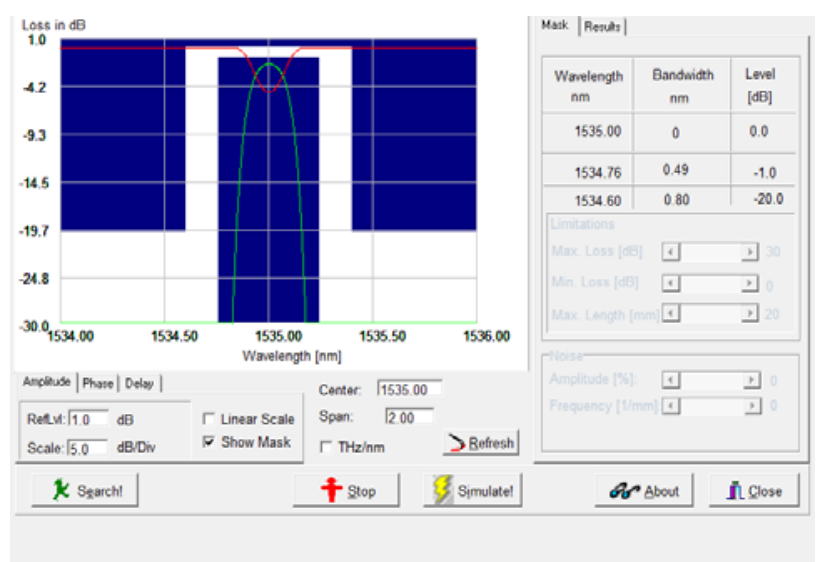

Fig. 5 Comparison of fiber Bragg gratings spectral characteristics without apodization - blue line and apodized Gauss function at $\mathrm{a}=3-$ red line

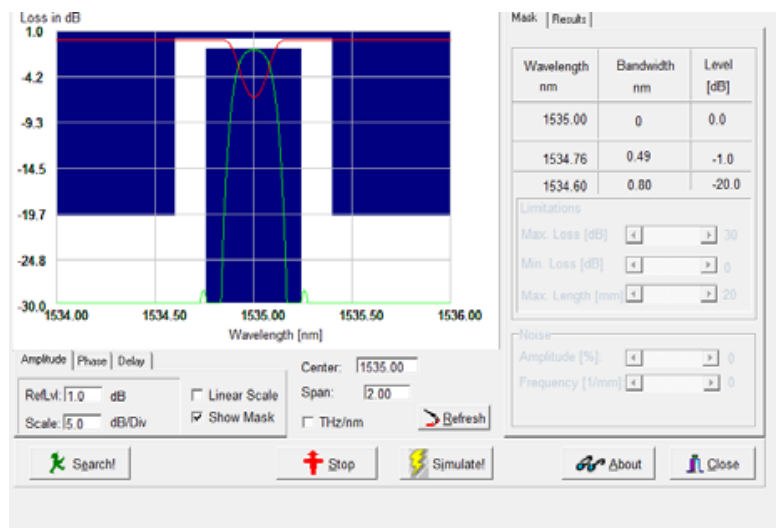

Fig. 6 Comparison of fiber Bragg gratings spectral characteristics without apodization - blue line and apodized Gauss function at $\mathrm{a}=5$ - red line.

Figures 7,8 and 9 show differences in fiber Bragg gratings spectral characteristics without apodization and with apodization using Gauss function with different values of parameter a. From the schemes thereof it is seen, that the number of side lobes decreases in apodized fiber Bragg gratings. Another widely used apodization function is cosine one. The function is expressed by the formula [10]:

$$
g(z)=\cos ^{A}\left(\frac{\pi}{L_{g}} z\right)
$$

where $\mathrm{A}$ is the function parameter. Examples of profiles for different values of the parameter $A$ are given in the figure 8 . 


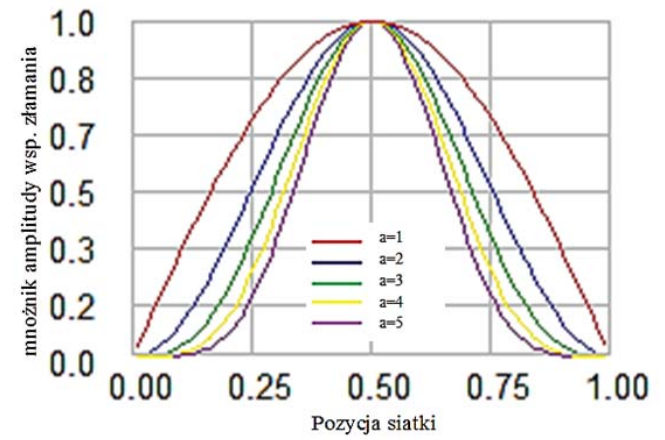

Fig. 7 - Cosine apodization function for different values of the parameter $\mathrm{A}$

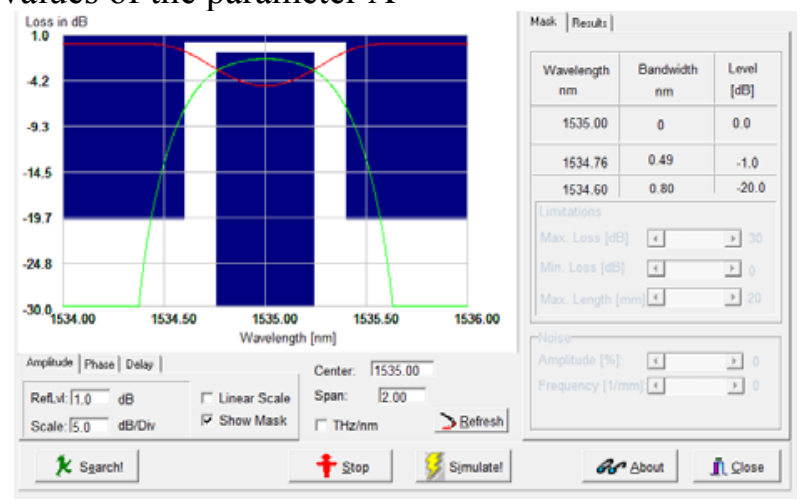

Fig. 8 Comparison of fiber Bragg gratings spectral characteristics without apodization - blue line and apodized cosine function for wsp. A $=2-$ red line

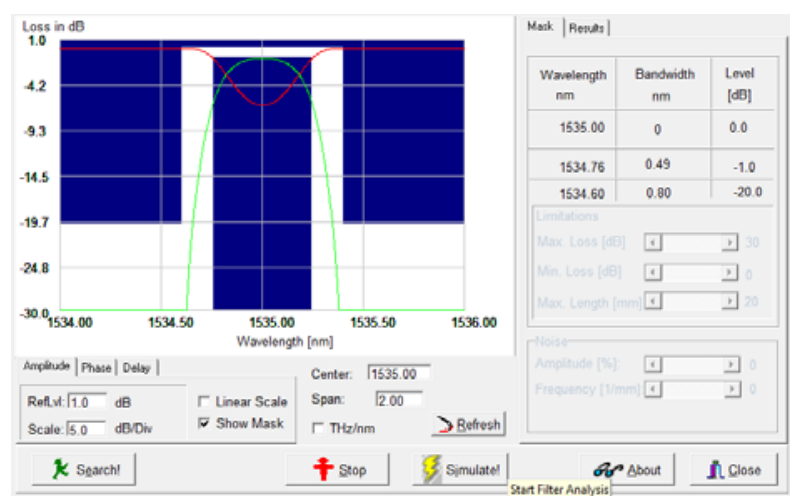

Fig.9 Comparison of fiber Bragg gratings spectral characteristics without apodization - blue line and apodized cosine function for wsp. A $=3$ - red line

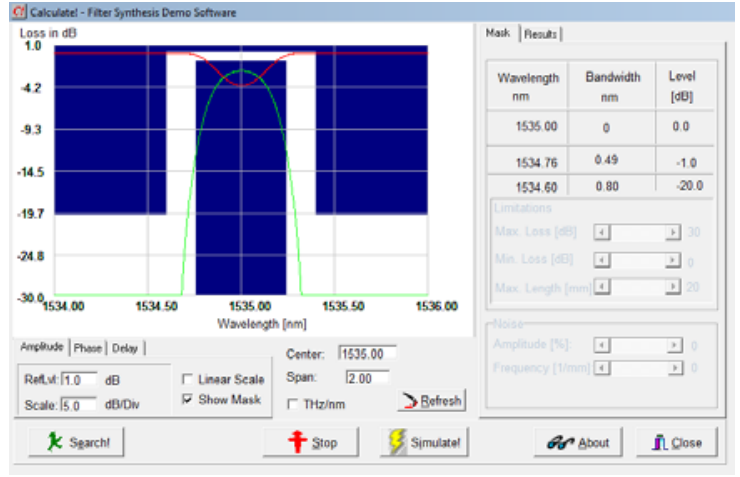

Fig. 10 Comparison of fiber Bragg gratings spectral characteristics without apodization - blue line and apodized cosine function for wsp. A = 5- red line

It is clearly seen from the charts that the side lobes are decreased in apodized gratings.

One more function is used for Bragg grating apodization, it is the so called Blackman function. The function is described by the formula [12]:

$g(z)=0,42+0,5 \cos \left(\frac{\pi z}{a}\right)+\frac{2}{25} \cos \left(\frac{2 \pi z}{a}\right)$,

where $a$ is the function's parameter. Figure 14 shows Blackman function for different values of the parameter $a$.

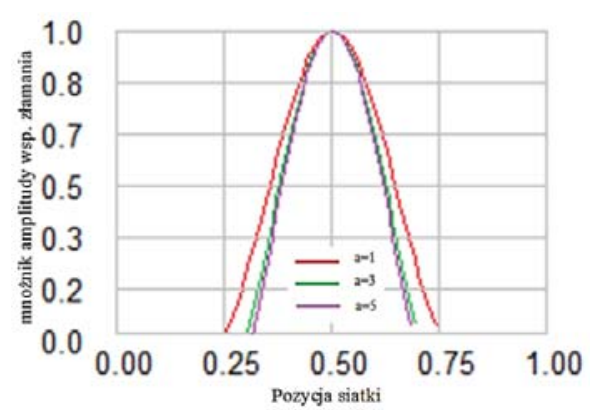

Fig. 11 Blackman apodization function for different values of the parameter $a$

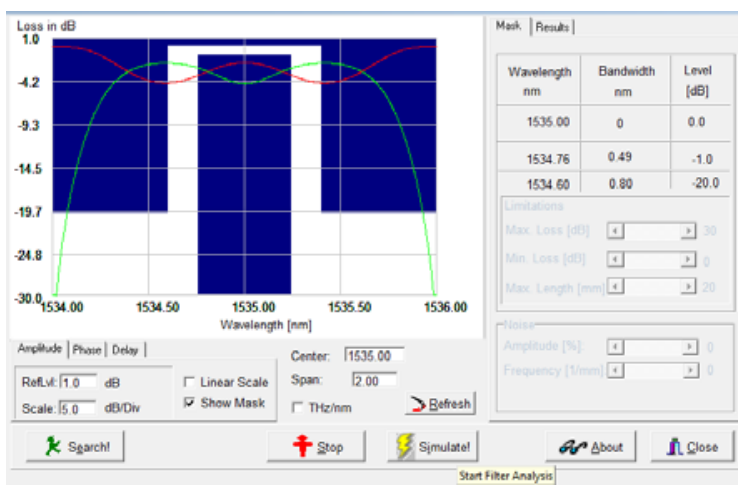


Fig. 12 Comparison of fiber Bragg gratings spectral characteristics without apodization - blue line and apodized Blackman function wsp. $a=2$ - red line

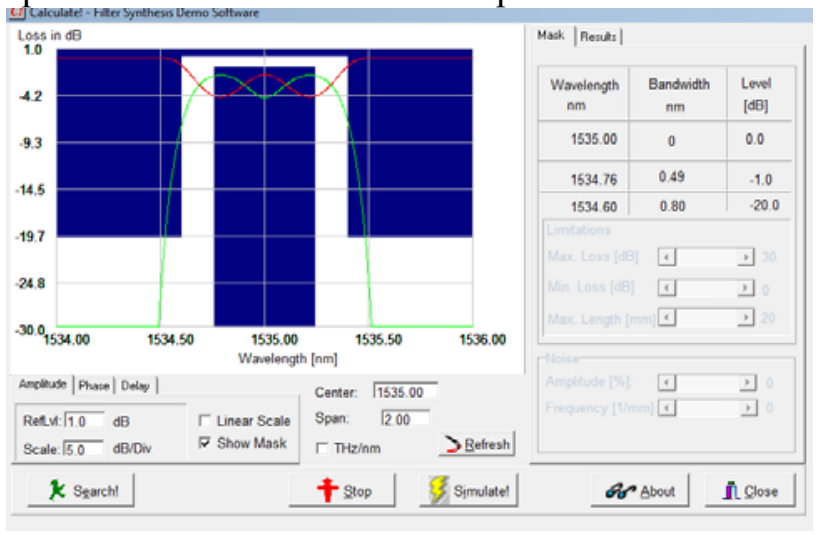

Fig. 13 - Comparison of fiber Bragg gratings spectral characteristics without apodization - blue line and apodized Blackman function wsp. $a=5-$ red line

In the figure 14 you can see, that Blackman function does not bring to big changes comparing to Gauss function. An important factor in case of applying Blackman apodization function is occurrence of local minimum, which breaks down the base peak into two parts. Charts 15 and 16 show that the value rise as well increases the importance of the minimum thereof.

One more is the sine function. This function differs from others by the fact that it has two parameters. The formula describes the function as follows [14]:

$$
g(z)=\operatorname{sinc}^{X}\left(\left|\frac{2(z-L / 2)}{L}\right|^{Y}\right),
$$

where $\mathrm{X}$ and $\mathrm{Y}$ are functional parameters.

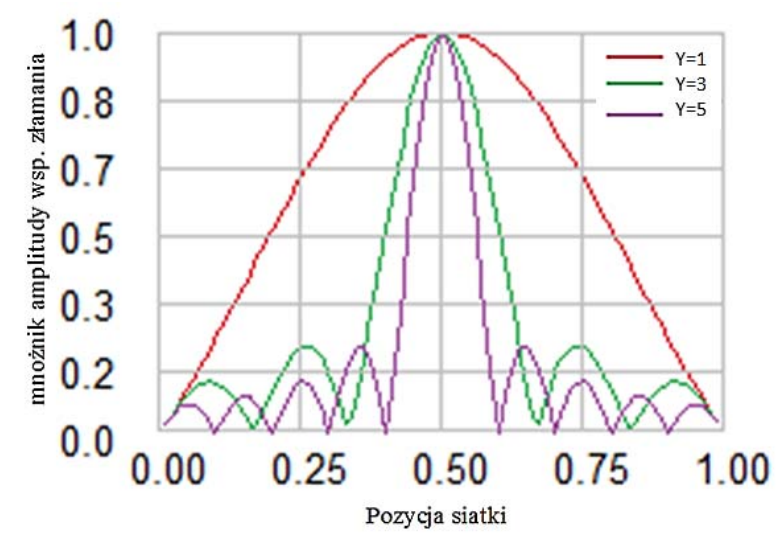

Fig. 14 Sine apodization function for different values of the parameter $Y$ at constant value $X=1$

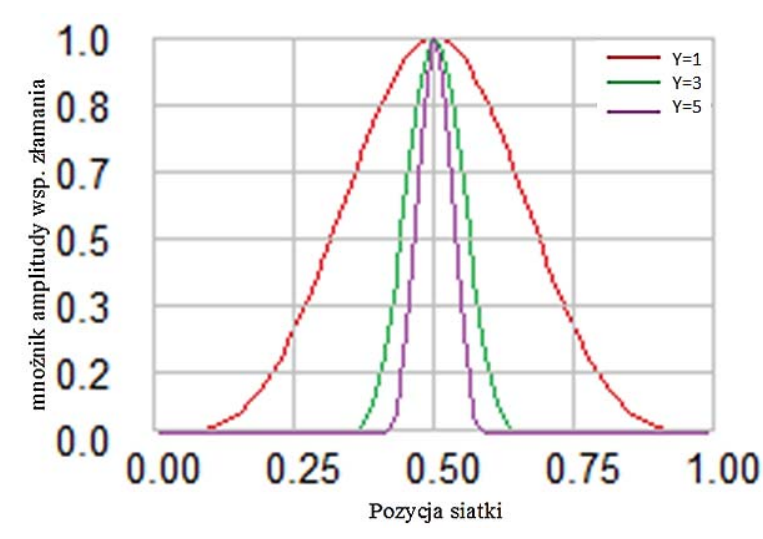

Fig. 15 Sine apodization function for different values of the parameter $Y$ at constant value $X=3$

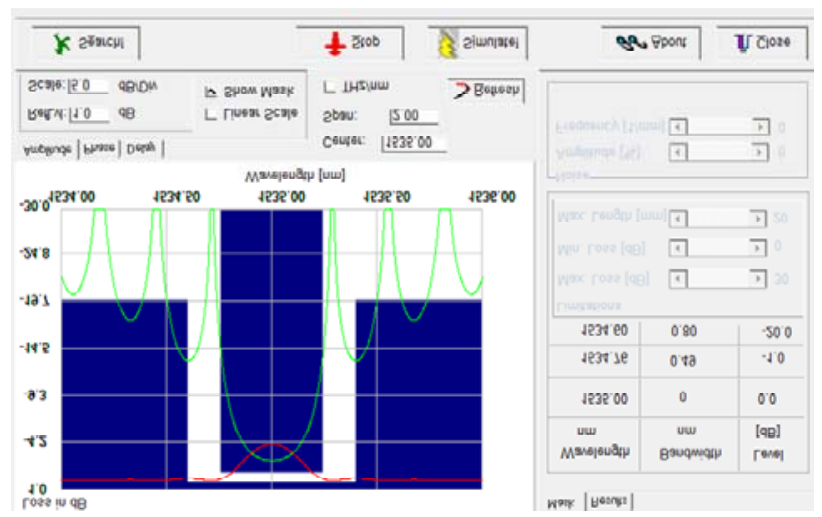

Fig. 16 Comparison of fiber Bragg gratings spectral characteristics without apodization - blue line and apodized sine function for wsp. $X=1$ and $Y=1-$ red line

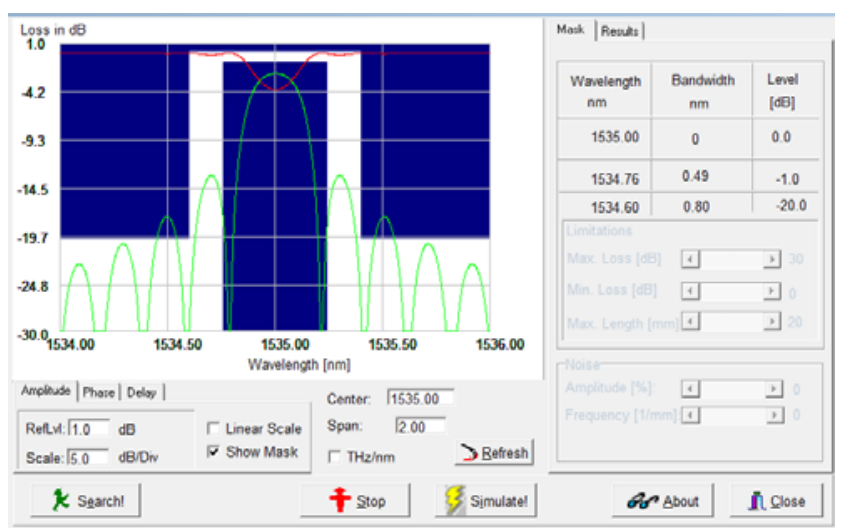

Fig. 17 Comparison of fiber Bragg gratings spectral characteristics without apodization blue line and apodized Blackman function for wsp. $\mathrm{X}=3$ and $\mathrm{Y}=1$-red line 


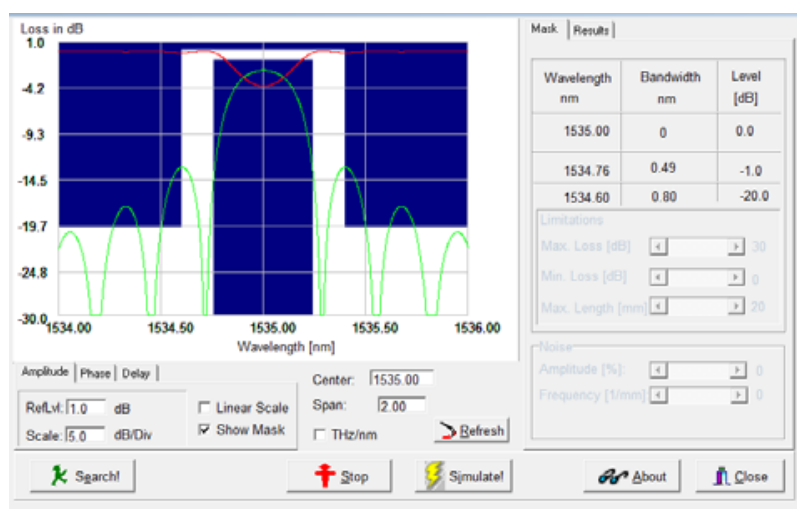

Fig. 18 Comparison of fiber Bragg gratings spectral characteristics without apodization blue line and apodized sine function for wsp. $\mathrm{X}=5$ and $\mathrm{Y}=1-$ red line

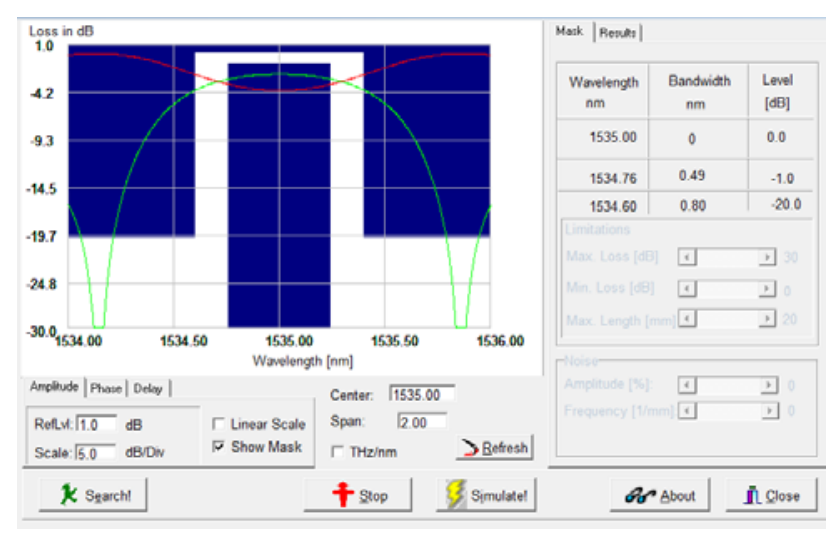

Fig. 19 Comparison of fiber Bragg gratings spectral characteristics without apodization - blue line and apodized sine function for wsp. $\mathrm{X}=1$ and $\mathrm{Y}=3-$ red line

Figures 17 and 18 show, that in a proper way, in virtue of $\mathrm{X}$ and $\mathrm{Y}$ parameters change, it is possible to get several apodization profiles. At the same time from spectral characteristics charts in the figures 20 - 22 it is seen, that the side lobes exclude the parameter increase, but they introduce noise characteristics. By contrast, with rising the parameter $Y$ the side lobes become a part of the base peak, which sufficiently distorts the grating characteristics.

\section{Conclusion}

Apodization function and its parameters proper selection have a great impact at reflective properties and Bragg fiber gratings dispersion compensation. Apodization is used to smooth the side straps of reflection spectrum, which leads to dispersion reduction, but it is less important, than the amplitude of the signal, reflected from the Bragg apodized gratings. Selection of the appropriate apodization function can substantially change the reflected light signal characteristics. Apodization function proper selection allow additional optimization of the grating features with account of its target application.

Fiber-optic sensors of physical quantity occupy an important place in sensor field due to the advantages which distinguish them from electronic analogues. A particular type is the sensor based on the Bragg fiber- optic gratings, which apart from having standard functions, characterizing the fiberoptic sensors, as well allow multiplexing, creating wide network, consisting of dozens sensors located in one optical fiber.

Applications multitude for the Bragg fiber gratings necessitates to influence at the grating optical spectrum nature. Upon temperature measuring the selection criterion is often consists in the most effective cutting the so-called side lobes, presence of which can influence much at sensor specifications, particularly upon using filter system interrogation. One of the techniques to eliminate the spectrum unfavorable features is apodization, commonly including the modulation of the refraction index depth changes along the structure. Applying the mathematical model, taking into account the apodizing function and creating the own simulation application, allows predicting the apodization parameters changes impact at the output spectrum. The examples show the influence of the selected input parameters at the output feature form.

This work is supported by grant from the Ministry of Education and Science of the Republic of Kazakhstan within the framework of the Project № AP05132778 «Research and development of signals interrogation system with fiber-optic refractometer in telecommunication networks».

\section{References:}

[1] Kashyap R. Fiber Bragg gratings, Academic Press, (1999) London.

[2] Vasiljyev S.A., Medvedkov O.I., Medvedkov I.G., Korolyev A.S., Bozhkov A.S., Kurkov Ye.M., Dianov (2005) Fiber gratings reflection index and their application, Quantum electronics, 35(12): 1085-1103.

[3] Hill K.O., Fujii F., Johnson D.C., Kawasaki B.S. (1978) Photosensitivity on optical fiber waveguides; application to reflection filter fabrication, Applied Physycs Letter, 32: 647-649.

[4] Kersey A.D., Davis M.A., Patrick H.J., LeBlanc M., Koo K.P., Askins C.G., Putnam M.A., 
Friebele E.J. (1997) Fiber grating sensors, Light wave Technology, 15(8): 1442-1463.

[5] Lopez-Higuera J.M. (2002) Handbook of optical fiber sensing technology, John Wiley \& sons, P. 795.

[6]Bilodeau F., Johnson D.C., Theriault S., Malo B., Albert J., Hill K.O. (1995) An all-fiber densewavelength multiplexer/DE multiplexer using photo imprinted Bragg gratings, IEEE Photonics Technology Letters, 7(4):388-390,

[7] Chai J., Yu Z., Liu Y. (2006) Analysis of the apodization parameter of linearly chirped Bragg gratings for dispersion compensation, School of Science, Beijing University of Posts and Telecommunications, Beijing.

[8]Zhang H. (2014) A novel method of optimal apodization selection for chirped fiber Bragg gratings, Optik 125, 1646- 1649.

[9] Khan S., Islam S. (2012) Determination of the Best Apodization Function and Grating Length of Linearly Chirped Fiber Bragg Grating for Dispersion Compensation, Bangladesh University of Engineering and Technology, Bangladesz.

[10] Wójcik W., Kashaganova G.B. (2015) Study of Bragg fiber gratings spectral characteristics, Materials of the II International scientificpractical conference «Information and telecommunication technologies: education, science, practice», Almaty, Kazakhstan, II vol., pp. 77-81.

[11] Kashaganova G.B. (2016) The influence of various apodization functions on the spectral characteristics of fiber Bragg gratings, Bulletin of KazNTU named after Satbayev K.I., 3(115):302311

[12] Li X., Chen X., Yin Y. and Xie S. (2003) A novel apodization technique of variable markspace ratio for fabricating sampled fiber bragg grating, Tsinghua University, China.

[13] Albert J. et al. (1995) Apodisation of the spectral response of fibre Bragg grat-ings using a phase mask with variable diffraction efficiency, Electronic Letters, 31: 222-223.

[14] Malo B., et al. (1995) Apodised in-fiber Bragg grating reflectors photoim- printed using a phase mask, Electronic Letters, 31: 223-225.

[15] Pastor D. et al. (1996) Design of apodized linearly chirped fiber gratings for dispersion compensation, J. Lightwave Technology, 14: 2581-2588.

[16] Keitmann-Curdes O., Brendel B., Marg C., Ermert H. (2002) Optimization of apodization based on the sidelobe pressure energy in simulated ultrasound fields, in: Proc. IEEE Int. Ultrason. Symp.,1677-1680.

[17] Abdullina S.R., Babin S.A., Kablukov S.I., Vlasov A.A. (2007) Simple technique of fiber Bragg gratings apodization by use Proc, Laser Optics 2006: Diode Lasers and Telecommunication Systems, ed. Nikolay N. Rosanov, 6612: 661201 (1-10).

[18] Chuang K.P. et al. (2004) Pure apodized phase-shifted fiber Bragg gratings fabricated by a two-beam interferometer with polarization control, IEEE Photonics Technology Letters, 16: 834-836.

[19] Othonos A., Kalli K. (1999) Fiber Bragg gratings. Artech House.

[20] Albert J., Hill K. O., Malo B. (1995) Apodization of spectral response of fiber Bragg gratings using phase mask with variable diffraction efficiency, Electronic Letters, 31(3): 222-223. 